\title{
Minimally Invasive Mitral Valve Surgery II Surgical Technique and Postoperative Management
}

\author{
J. Alan Wolfe, MD, * S. Chris Malaisrie, MD, $\uparrow$. Saeid Farivar, MD, PhD, $\neq$ Junaid H. Khan, MD, $\S$ \\ W. Clark Hargrove, MD,// Michael G. Moront, MD, 9 William H. Ryan, MD, \# Gorav Ailawadi, MD, ** \\ Arvind K. Agnihotri, MD, $†$ Brian W. Hummel, MD, $\neq \neq$ Trevor M. Fayers, MD, $\$ \oint$ Eugene A. Grossi, MD,/// \\ T. Sloane Guy, MD, 99 Eric J. Lehr, MD, PhD, \#\# John R. Mehall, MD, *** Douglas A. Murphy, MD, $\uparrow \uparrow$ \\ Evelio Rodriguez, MD, f+ Arash Salemi, MD, $\$ \oint$ Romualdo J. Segurola, Jr, MD,//I/I \\ Richard J. Shemin, MD, 199 J. Michael Smith, MD, \#\#\# Robert L. Smith, MD, \# Paul W. Weldner, MD, **** \\ Clifton T. P. Lewis, MD, ††† Glenn R. Barnhart, MD,\#\# and Scott M. Goldman, MD
}

\begin{abstract}
Techniques for minimally invasive mitral valve repair and replacement continue to evolve. This expert opinion, the second of a 3-part series, outlines current best practices for nonrobotic, minimally
\end{abstract}

Accepted for publication June 16, 2016.

From the *Northeast Georgia Physicians Group, Gainesville, GA USA $†$ Northwestern University, Feinberg School of Medicine, Chicago, IL USA; $\$$ Minneapolis Heart Institute, Abbott Northwestern Hospital, Minneapolis, MN USA; §East Bay Cardiac Surgery Center, Oakland, CA USA; \#Penn Presbyterian Medical Center, Philadelphia, PA USA; ๆThe Toledo Hospital, Toledo, OH USA; \#The Heart Hospital Baylor Plano, Plano, TX USA; **University of Virginia, Charlottesville, VA USA; ††Saint Elizabeth's Medical Center, Brighton, MA USA; \$Gulf Coast Cardiothoracic \& Vascular Surgeons, Ft. Myers, FL USA; §§Holy Spirit Northside Hospital, Chermside, Australia; ||N|New York University School of Medicine, New York, NY USA IqTemple University, Philadelphia, PA USA; \#\#Swedish Heart and Vascular Institute, Seattle, WA USA; ***Penrose St. Francis Hospital, Colorado Springs, CO USA; †††Emory St. Joseph's Hospital, Atlanta, GA USA; +†tSt. Thomas Hospital, Nashville, TN USA; $\S \S$ Weill Cornell Medical College/New York Presbyterian Hospital, New York, NY USA; ||||||South Florida Heart \& Lung Institute, Doral, FL USA; 9 qDavid Geffen School of Medicine, University of California Los Angeles, Los Angeles, CA USA; \#\#TriHealth Heart Institute, Cincinnati, OH USA; ****Central Maine Heart and Vascular Institute, Lewiston, ME USA; ††† Baptist Hospital, Birmingham, AL USA; and H†tLankenau Medical Center, Wynnewood, PA USA.

Eugene A. Grossi, MD, is now at Cornell Wiel Medical School, New York, NY USA.

Supported by Princeton Baptist Hospital, Birmingham, AL USA, which provided funds for medical editing assistance, which was performed by Jeanne McAdara-Berkowitz, PhD. The funds came from an educational budget and were not provided by any commercial entity. Medical illustrations created by Jill Rhead, MA, CMI, FAMI, were donated by Edwards Lifesciences Corp, Irvine, CA USA

Disclosures: See next page.

Address correspondence and reprint requests to Scott M. Goldman, MD, Lankenau Hospital, 280 Lankenau MSB, 100 Lancaster Ave, Wynnewood, PA 19096 USA. E-mail: GoldmanS@mlhs.org.

Copyright (C) 2016 by the International Society for Minimally Invasive Cardiothoracic Surgery

This is an open-access article distributed under the terms of the Creative Commons Attribution-Non Commercial-No Derivatives License 4.0 (CCBY-NC-ND), where it is permissible to download and share the work provided it is properly cited. The work cannot be changed in any way or used commercially.

ISSN: 1556-9845/16/1104-0251 invasive mitral valve procedures, and for postoperative care after minimally invasive mitral valve surgery.

Key Words: Minimally invasive surgery (includes port access, minithoracotomy), Mitral valve, repair, replacement, Surgery/incisions/ exposure/techniques, MVR, MIMVR, Heart valve, Thoracotomy, Endoscopic, Lower hemisternotomy.

(Innovations 2016;11: 251-259)

N inimally invasive mitral valve (MV) repair and replacement (MIMVR) was first described as a potential alternative to full-sternotomy MV surgery in the mid-1990s. ${ }^{1}$ Since that time, 4 techniques have emerged as acceptable approaches to MIMVR: lower hemisternotomy, direct-vision right minithoracotomy, endoscopic right minithoracotomy, and robotic-assisted right minithoracotomy.

\section{See accompanying editorial on page 233 , and articles on pages 243 and 260}

No randomized clinical trials have yet been undertaken to directly compare the safety and effectiveness of minimally invasive approaches with each other or full sternotomy. Thus, there have, to date, been minimal data to guide practicing surgeons and few descriptions of standardized techniques.

Recognizing that the field of MIMVR has matured such that greater standardization is desirable, a group of 26 US surgeons with combined experience of approximately 17,000 MIMVR operations convened a working group to compile initial guidance based on expert consensus. The group does not propose to either establish the superior efficacy or safety of one MIMVR approach over another, or to compare them to a fullsternotomy approach; instead, the goal was to describe a stepwise approach for surgeons wishing to gain new knowledge about current MIMVR approaches.

The present report is the second of a 3-part series of articles that are intended to be read together. This current work focuses on the 3 nonrobotic operative approaches to MIMVR. Patient selection and evaluation, and preoperative planning for successful cannulation, systemic perfusion, myocardial protection, and anesthetic preparation, were addressed in the first part 


\begin{abstract}
Disclosures: All authors declare that they serve as consultants on the Medical Advisory Board for Edwards Lifesciences Corp, Irvine, CA USA. In addition, S. Chris Malaisrie, MD, is a consultant for Medtronic, Inc, Minneapolis MN USA, and Abbott Vascular, Abbott Park, IL USA. R. Saeid Farivar, $\mathrm{MD}, \mathrm{PhD}$, is a consultant to Abbott Vascular, Abbott Park, IL USA, and Medtronic, Inc, Minneapolis, MN USA. W. Clark Hargrove, MD, was a consultant to Sorin/LivaNova, Milan, Italy. Michael G. Moront, MD, is a consultant for Medtronic Heart Valves, Medtronic, Inc, Minneapolis, MN USA, Terumo Cardiovascular Group, Ann Arbor, MI USA, and LSI SOLUTIONS, Victor, NY USA. William H. Ryan, MD, is a consultant to Medtronic, Inc, Minneapolis, MN USA. Gorav Ailawadi, MD, is a consultant to Abbott Vascular, Ab bott Park, IL USA, AtriCure, Inc, West Chester, OH USA, Medtronic Inc, Minneapolis, MN USA, and St. Jude Medical, Inc, St. Paul, MN USA. Brian W. Hummel, MD, is a consultant to AtriCure, Inc, West Chester, $\mathrm{OH}$ USA, and Medtronic, Inc, Minneapolis, MN USA. Eugene A. Grossi, $\mathrm{MD}$, is a consultant to Medtronic, Inc, Minneapolis, MN USA, and Intuitive Surgical, Sunnyvale, CA USA. T. Sloane Guy, MD, is a consultant to Medtronic Inc, Minneapolis, MN USA, and Ethicon Inc/Johnson\&Johnson, Somerville, NJ USA, and a recipient of a grant from Biomet, Inc, Warsaw, IN USA. John R. Mehall, MD, is a consultant to AtriCure, Inc, West Chester, $\mathrm{OH}$ USA. Douglas A. Murphy, MD, receives product royalties from Medtronic, Inc, Minneapolis, MN USA. Evelio Rodriguez, MD, is a consultant to Abbott Vascular, Abbott Park, IL USA, AtriCure, Inc, West Chester, $\mathrm{OH}$ USA, and St. Jude Medical, Inc, St. Paul, MN USA, is a grant recipient from Medtronic, Inc, Minneapolis, MN USA, and serves on the speaker's bureaus for Edwards Lifesciences, Corp, Irvine, CA USA, and Medtronic, Inc, Minneapolis, MN USA. Arash Salemi, MD, is a consultant to Medtronic, Inc, Minneapolis, MN USA. J. Michael Smith, MD, is a consultant to AtriCure, Inc, West Chester, OH USA, and Intuitive Surgical, Sunnyvale, CA USA. Robert L. Smith, MD, serves on the speaker's bureaus for Abbott Vascular, Abbott Park, IL USA, and Intuitive Surgical, Inc, Sunnyvale, CA USA. Clifton T. P. Lewis, MD, is a consultant to Intuitive Surgical, Inc, Sunnyvale, CA USA. Glenn R. Barnhart, MD, is a consultant to AtriCure, Inc, Wes Chester, OH USA, and On-X Life Technologies, Austin, TX USA. Scott M. Goldman, MD, serves on advisory boards for Abbott Vascular, Abbott Park, IL USA, and Medtronic, Inc, Minneapolis, MN USA, and is a consultant to St. Jude, Inc, St. Paul, MN USA, and LSI SOLUTIONS, Victor, NY USA. J. Alan Wolfe, MD, Junaid H. Khan, MD, Arvind K. Agnihotri, MD, Trevor M. Fayers, MD, Eric J. Lehr, MD, PhD, Romualdo J. Segurola, Jr, MD, Richard J. Shemin, MD, and Paul W. Weldner, MD, declare no other conflicts of interest.
\end{abstract}

of the series. ${ }^{2}$ The third report will describe considerations specific to robotic-assisted MIMVR, as well as training pathways that will prepare the surgical team to move from fullsternotomy to minithoracotomy to robotic approaches. ${ }^{3}$

\section{FIRST PRINCIPLES OF CARDIAC SURGERY AND MIMVR}

As with any cardiac operation, MIMVR is based on 4 fundamental tenets: (1) establishment and maintenance of adequate cannulation and perfusion, (2) complete myocardial protection, (3) optimal exposure, and (4) procedures appropriate to the patient's specific pathology. ${ }^{4}$ A methodical approach to performing each step within the overall MIMVR is a key concept. The consequences of poorly conducted cardiopulmonary bypass (CPB), failure to confirm adequate venous drainage before cardiotomy, inadequate myocardial protection, and suboptimal surgical exposure are magnified in MIMVR. Each step must be completed before proceeding to the next to avoid extending operative times or otherwise compromising the operation. Facilitating the application of these principles, use of an efficient stepwise procedural approach, and maintenance of open team communication are essential to success.

Since most MIMVRs are not done with central cannulation for CPB, familiarity with the techniques of peripheral cannulation and thorough preoperative planning form the basis of a safe procedure. ${ }^{2}$ With only basic endovascular wire skills, the surgeon should establish CPB with the goal of complete unloading of the heart and reliable systemic perfusion. The consequences of poor CPB are compromised surgical exposure, poor myocardial protection, and malperfusion syndrome.

When transitioning from full sternotomy to MIMVR, a dependable method of myocardial protection is necessary. ${ }^{2}$ Longer periods of myocardial ischemia are required for MIMVR, and consequences of poor myocardial protection are right-sided heart dysfunction or even biventricular failure. Rarely is a strategy depending on ventricular fibrillation without aortic cross clamping used as a sole means of protection.

Surgical exposure of the MV in mini-thoracotomy approaches can be equal to or better than sternotomy approaches. Exposure is predicated upon appropriate placement of the working incision in relation to the pulmonary hilum and greatly facilitated using endoscopy. During the transition to MIMVR, it is helpful to be familiar with the use of enabling technologies, some of which may also be used in the sternotomy approach. Long-shafted instruments, auto-knotting devices, transthoracic aortic cross clamps, and endoaortic occlusion balloons not only facilitate the procedure but also improve visibility and exposure.

As with all cardiac surgery, a team that is familiar with minimally invasive techniques and technology will facilitate safe conduct of MIMVR. It is important that the team is briefed on the operative plan for each minimally invasive valve procedure, and that communication among the team members is established and encouraged. Members of the team should be empowered to voice any concerns.

\section{SPECIFIC SURGICAL APPROACHES TO MIMVR}

The 3 approaches described here roughly follow a natural progression in the development of skills necessary to moving from a full sternotomy to a minithoracotomy approach. ${ }^{5,6}$

\section{Lower Hemisternotomy Technique}

Lower hemisternotomy is an option for MIMVR as well as atrial septal defect closure, tricuspid valve repair or replacement, and atrial fibrillation ablation with left atrial appendage closure. ${ }^{7}$ Limited coronary artery bypass grafting is also possible. The key advantage of this approach is the ability to directly cannulate and cross-clamp the aorta. Morbid obesity is a relative contraindication, and this approach is contraindicated in reoperations because of the difficulty that may be encountered in exposing the aorta.

The operative strategy, setup, and anesthesia protocols are similar to full-sternotomy MV procedures, with a few modifications. Defibrillator pads are placed on the patient before surgery. The angle of Louis (sternomanubrial junction) marks the second rib, and a $6-$ to $8-\mathrm{cm}$ vertical skin incision is made in the midline, starting $2.5 \mathrm{~cm}$ caudal to the angle of Louis toward the xyphoid process (Fig. 1). A reciprocating saw, with the blade pointing up, is used to enter the right-sided attachments of the xiphoid process and a sternotomy is made cephalad toward the angle of Louis. The sternotomy is extended into the right second intercostal space and can be performed by gently curving the reciprocating saw or using an oscillating saw with a narrow blade. The manubrium is kept intact while maintaining adequate exposure from the second intercostal space to the xiphoid process. Care is taken not to injure the right internal mammary (thoracic) artery or vein. Owing to the small skin incision, a standard sternal retractor cannot be placed. A variety of low-profile sternal retractors with narrow blades 


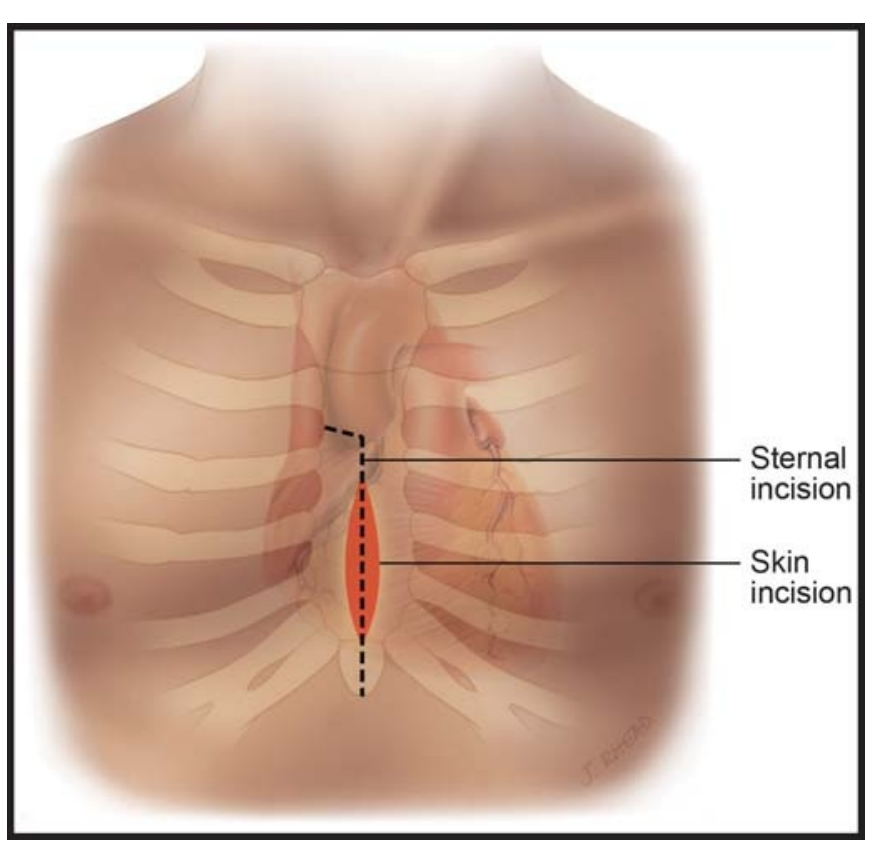

FIGURE 1. Lower hemisternotomy incision.

and attachments for self-retaining left-atrial retractors are available and are recommended. Carbon dioxide $\left(\mathrm{CO}_{2}\right)$ is routinely flooded into the operating field to assist in de-airing of the heart at the end of the procedure.

The pericardium is opened vertically, and since the manubrium is left intact, the operative field may be moved caudad to improve exposure. Tonsil clamps are used to grasp the pericardium and pull it caudad. Three silk sutures are placed on the right side of the pericardium, with a skew toward the cranial half so as to pull the pericardium caudally. The needles are kept on the suture, and the retractor is removed. The sutures are then sewn on the caudal half of the skin incision and pulled up tightly. The retractor is then re-replaced and opened. This exposes the heart and pulls the aorta caudally into the incision (Fig. 2).

A double purse-string is placed in the ascending aorta. A tonsil clamp can grasp the fat pad to assist retraction. A standard ascending aortic cannula is then placed and secured to the skin. Venous cannulation and drainage is typically approached femorally, either percutaneously or by cutdown, and is augmented as needed by the addition of a neck superior vena cava (SVC) cannula. A root vent is placed into the ascending aorta for de-airing and antegrade cardioplegia. A retrograde cardioplegia cannula can be placed according to the surgeon's preference. ${ }^{2}$

Once CPB is initiated, mild hypothermia is instituted. Vacuum or kinetic-assist venous drainage augments emptying of the heart. Aortic occlusion can be accomplished by placing an aortic cross clamp in the standard manner. The aortopulmonary junction is not opened, and a left atrial vent is not used. Once the clamp is placed, antegrade cardioplegia is given. The interatrial groove is developed, and the right atrium can be freed from the left atrium (LA) up to the fossa ovalis. The LA is widely opened, from the SVC to the oblique fossa under the inferior vena cava, to facilitate exposure of the MV.

Mitral valve repair or replacement techniques are standard per surgeon's preference. After completion of the MV surgery, closure of the LA, de-airing, decannulation, placement of chest tubes, and temporary epicardial pacing wires are performed in the standard manner. Typically, 2 soft chest drains are placed, with one placed into the oblique sinus and the other anteriorly.

Closure is typically performed with 4 simple wires, completely encircling the sternum at the intercostal spaces. At the junction at the second intercostal space, a wire is placed at a 45-degree angle to ensure vertical stability and minimize postoperative sternal clicking. Alternatively, a sternal plate can be used to secure the entire sternum.

\section{Direct-Vision Right Minithoracotomy Technique}

A right lateral approach is an attractive option for MIMVR because it causes less surgical trauma compared to sternotomy, allowing patients to return to functional status within 2 weeks instead of 1 to 2 months. ${ }^{8}$ Other advantages include "straight-on" visualization of the MV, decreased blood loss, lower incidence of significant wound infections, and improved cosmesis. ${ }^{9-14}$

Anesthesia protocol is generally the same as in conventional MV repair. Earlier extubation can be accomplished by modifying the anesthetic agents being used. Single-lung ventilation using either a double-lumen tube or a bronchial blocker greatly facilitates the procedure. Before starting $\mathrm{CPB}$, the right lung can be deflated to identify landmarks. In reoperations, lung adhesions can be easily divided. Finally, hemostasis is facilitated at the end of the procedure with the lung deflated. Some surgeons prefer not to use single-lung ventilation; however, this technique comes at the expense of increased CPB times.

Bilateral arterial lines are required when using endoaortic balloon occlusion. Care should be taken to avoid dampening these tracings, and monitors should be set to ensure that these tracings overlap with those for the root vent. Transvenous placement of a pulmonary vent, or addition of an SVC cannula into the venous circuit using a Y connector, may significantly aid in drainage

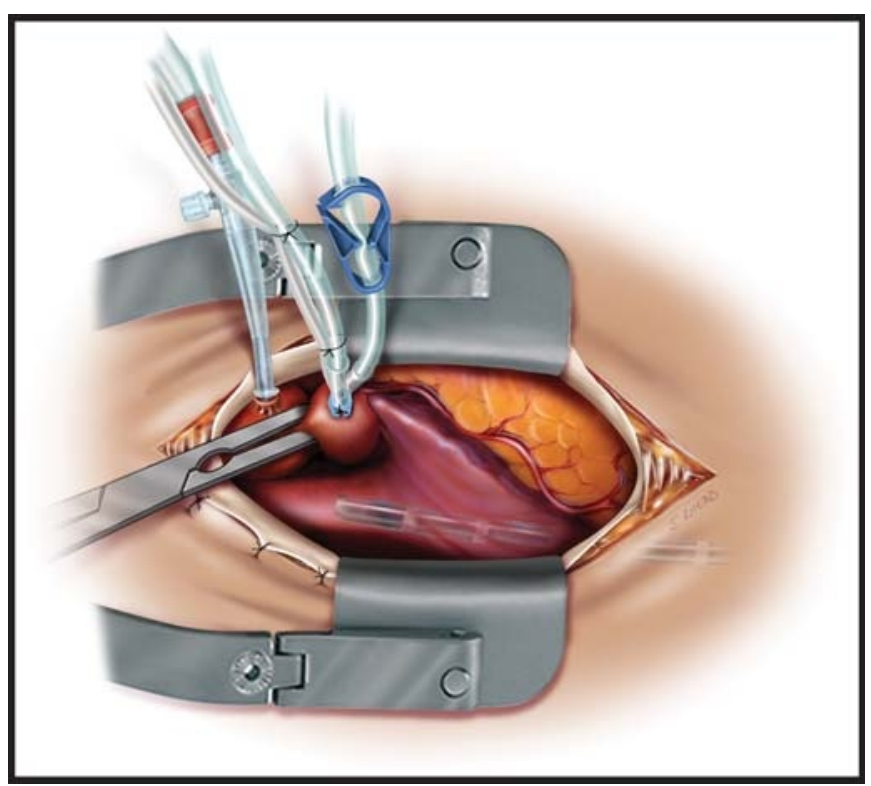

FIGURE 2. Lower hemisternotomy showing placement of retractor and cannulae. 
(discussed later in the text). Significant variations exist in the use of retrograde cardioplegia; some experienced centers use it in every case, others not at all. In the case of mild to moderate aortic insufficiency and reoperative cases, retrograde cardioplegia can be beneficial, and it can be placed either directly through the right atrial free wall or percutaneously through the right internal jugular vein.

The patient is positioned supine (Fig. 3) all the way to the right side of the table, with the right arm fully supported by the table and slightly flexed to improve access to the anterior axillary line. A small pillow or IV bag is placed inferior to the scapula to open up the axillary space. The head should remain in the midline and should be supported to minimize risk of brachial plexus injury. Placement of defibrillator pads across the chest wall is routine, as access to the ventricles is limited. A plastic adhesive incise drape is helpful in retracting breast tissue superiorly and medially to facilitate exposure and "relocate" the inframammary crease to the appropriate intercostal space.

The primary incision is placed at the level of the hilum, usually in the fourth intercostal space, starting at the midclavicular line and extending laterally to the anterior axillary line. Large incisions can be used in the beginning, becoming smaller and more lateral with greater experience. The incision should be large enough to allow for adequate light to reach the MV. A head lamp may be useful with this technique.

Next, attention is directed to chest wall hemostasis. Placement of a clean lap tape can help identify troublesome spots. A soft tissue retractor is placed, and slight rib spreading may be required. Carbon dioxide insufflation, which is useful in minimizing air in minithoracotomy as well as endoscopic cases, is established at 2 to $3 \mathrm{~L} / \mathrm{min}$.

An incision parallel to the inguinal ligament exposes the common femoral vessels. Intraoperative ultrasound can facilitate a smaller incision in the groin and help to identify the ideal position on the vessel proximal to the profunda femoris. Using only electrocautery for the dissection and avoiding injury to the tissue between the artery and vein will help prevent the development of a lymphocele.

Venous cannula placement is carried out over a wire with guidance from transesophageal echocardiogram (TEE). The vein is accessed with an 18-gauge needle, and a J-tip wire is passed to the right side of the heart. The wire can be seen on a bicaval echocardiographic view, although with additional lines traversing the right atrium, caution must be taken that misidentification does not occur. The J-tip should be visualized by all team members as it traverses the right atrium into the SVC. The tapered introducer protrudes from the line by several centimeters, and the cannula is gently advanced this distance as the introducer is removed. The TEE appearance of the venous cannula will not be readily apparent until the introducer is removed; the typical "railroad track" appearance will confirm optimal position in the SVC. The end of the cannula should be positioned 2 to $3 \mathrm{~cm}$ above the atrial-SVC junction to insure adequate brachiocephalic drainage if it is retracted by subsequent atrial septal "lifting" for valve exposure.

When the occasion arises, a percutaneous modified Seldinger technique can be used to gain access to the femoral vein. Care must be taken to avoid bending the J-tip wire, which could result in poor track development, especially in obese patients. Exchanging the J-tip wire with a stiff guidewire can be used to avoid this problem. Upon removal, a deep skin stitch, such as a horizontal mattress or figure-of-eight using a 2-0 suture, is all that is necessary for closure with removal of the suture on day 3 or day 4 .

No operation should proceed in the presence of inadequate venous drainage. Before anticipated aortic clamping, complete decompression of the right atrium must be confirmed by a low transduced central venous pressure, a nonpulsatile arterial waveform, and direct visualization of the right atrial chamber. Vacuum-assisted suction is used for venous drainage. In general, the amount of suction should not exceed $40 \mathrm{~mm} \mathrm{Hg}$. If adequate decompression of the right atrium is not possible with addition of vacuum, the venous cannula is repositioned. The use of a

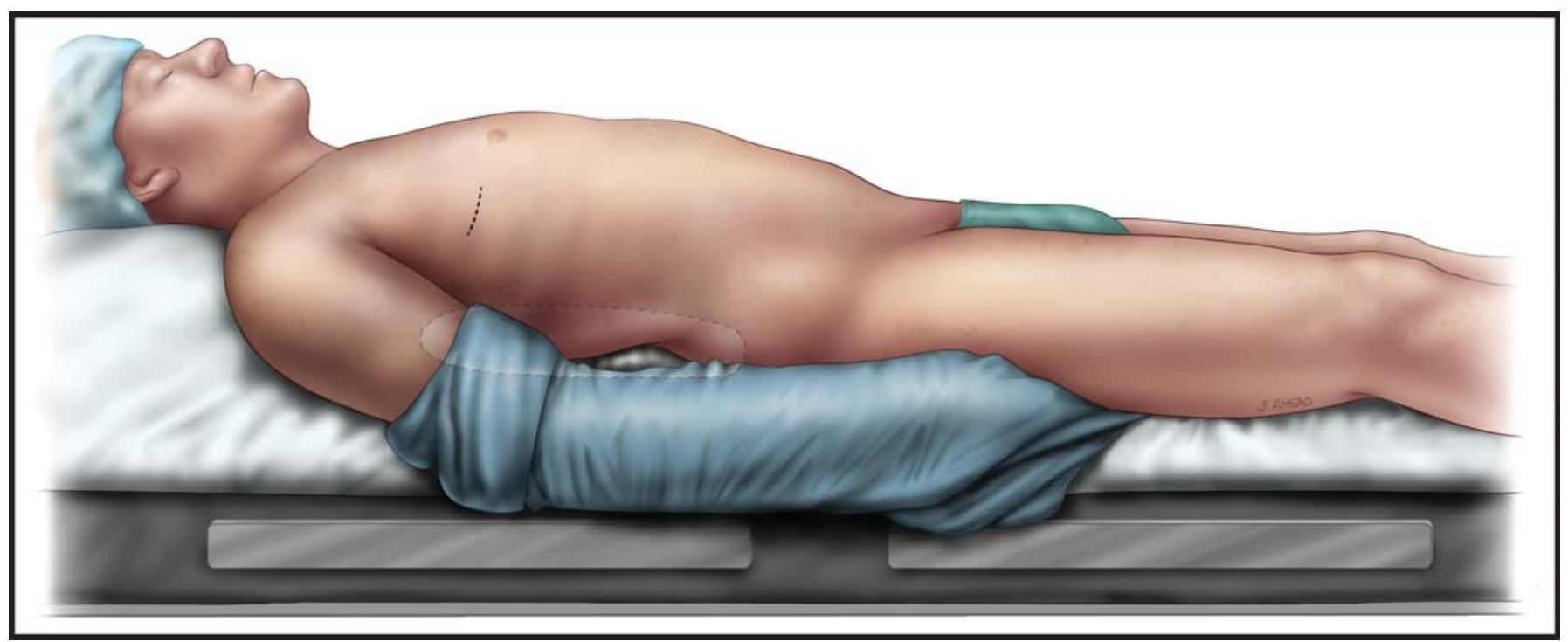

FIGURE 3. Patient positioning for direct-vision right minithoracotomy, showing a small pillow placed inferior to the scapula (transparent ellipse). The vertical dashed line indicates the placement of the primary incision. 
multiside-hole venous cannula can reduce the need to reposition and may provide improved drainage.

Drainage can deteriorate during the conduct of the operation. This typically occurs during movement of the left atrial retractor, which can interfere with the drainage from the upper body. If a distended SVC can be visualized, either a different angle of retraction or repositioning of the cannula is needed. Drainage can also be poor in larger patients when an SVC cannula is not used, despite all efforts to remediate. In that setting, it is prudent to place an additional thoracic trocar and directly cannulate the SVC with a separate, wire-reinforced, 24-F cannula and connected into the venous circuit with a Y connector.

After visual confirmation that the right side of the heart is empty and there is no cardiac ejection, the pericardium is opened and stay sutures are placed. A figure-of-eight suture or pledgeted horizontal mattress suture using 0 silk is placed at the superior portion of the dome of the diaphragm and passed through the chest wall inferiorly to allow for gentle caudal retraction (Fig. 4). This "flattening" of the diaphragm allows for complete direct visualization from the costophrenic pericardial reflection to the level of the mid ascending aorta. Visual observation and attention to individual anatomical differences must be exercised to avoid excessive tension on the right phrenic nerve.

Direct aortic cross clamping or endoaortic balloon occlusion is followed by antegrade cardioplegia and retrograde cardioplegia, if used. The oblique sinus is widely entered posterior to the inferior vena cava. It is important that the incision be carried far enough inferiorly to "release" the posterior atrial wall and prevent posterior atrial wall "tenting." Turning the pulmonary artery vent off will facilitate entering the LA. The atrial retractor post is placed through a separate entry in the same intercostal space as the working port, as medial as possible, avoiding injury to the internal mammary artery. The atrial retractor blade is chosen based on the size and depth of the LA to achieve superior visualization. The blade should sit near the posteromedial commissure. The atrial retractor post is retracted anteriorly and connected to an external retractor stabilization device. If MV visualization is not ideal, repositioning of the retractor post and expansion of the working port should be achieved before initiating repair.

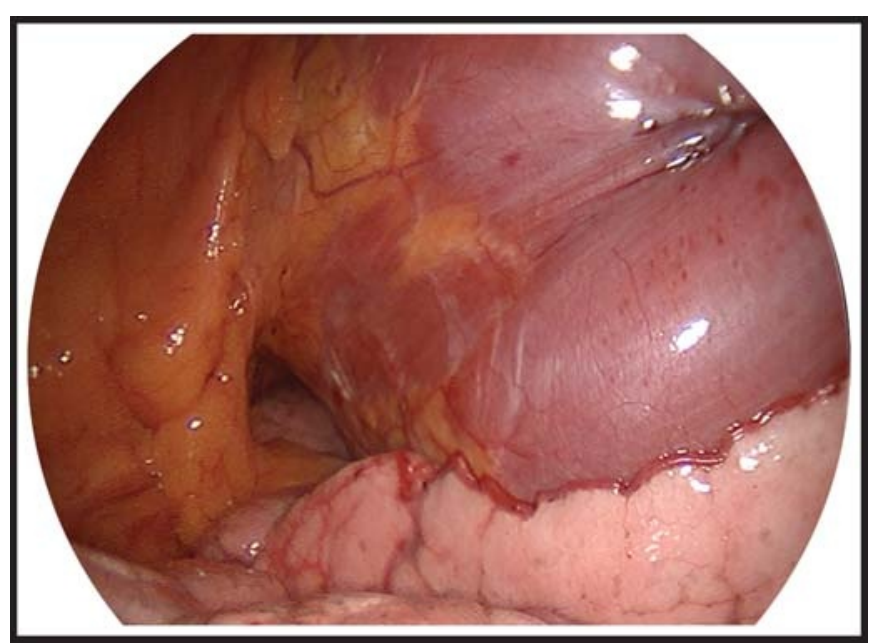

FIGURE 4. Exteriorization of sutures for diaphragmatic retraction.
Mitral valve repair or replacement techniques are standard according to surgeon's preference. Shafted instruments and use of an autoknotting device will facilitate the procedure. Additional procedures may be also performed such as atrial fibrillation ablation, left atrial appendage closure, and tricuspid valve repair according to surgeon preference, keeping in mind that concurrent tricuspid valve repair requires bicaval cannulation, and the addition of concurrent procedures will lengthen already prolonged clamp times. These may be added after the team obtains significant experience with isolated primary MV operations.

Pacing wires should be placed on the inferior wall of the right ventricle before removing the clamp or other occlusion device. In reoperative cases, a Swan-Ganz (Edwards, Irvine, CA USA) catheter or previously placed transvenous pacing wire is required.

Routine use of $\mathrm{CO}_{2}$ minimizes residual intracardiac air. ${ }^{15}$ De-airing is accomplished by filling the heart while closing the atriotomy and by sumping the left ventricle. Gentle suction is placed on the aortic vent or the central lumen of the endoaortic balloon catheter as appropriate. The clamp is removed, and the heart is allowed to recover. Cardiopulmonary bypass is discontinued.

Closure of the pericardium may be performed by either loosely applying sutures, or with a patch. One soft chest drain is left in the pericardium, and a second should be left in the pleural space. The ribs should be loosely approximated to prevent lung herniation. Before closure, the chest wall should be examined meticulously for bleeding; an angled mirror or scope can be helpful for looking at the port, retractor, and stay suture sites.

\section{Endoscopic Right Minithoracotomy Technique}

Adoption of an "endoscopic" approach to port access surgery allows full expression of the surgical skill sets one has developed during the progression from sternotomy to a minimally invasive approach. ${ }^{5,6}$ As such, it should be noted that a number of the technique-related suggestions presented here may also be useful to surgeons performing direct-vision minithoracotomy.

The term endoscopic refers to the intracardiac portion of the procedure, which is performed through the smallest possible working incision, ideally requiring a soft tissue retractor and minimal to no rib spreading. The extracardiac portion of the operation, in both exposure and atrial closure, can be performed endoscopically, but these steps are more easily accomplished under direct vision through the working port. This approach allows for increased accuracy and efficiency during the preparatory and closing portions of the procedure while ultimately providing unobstructed visualization of the valve.

The working incision is usually placed in the fourth or fifth intercostal space at the anterior axillary line and extended $4 \mathrm{~cm}$ laterally (Fig. 5). This allows for subsequent insertion of a 35- to 45-mm atrial retractor "blade," and will admit 1 or 2 fingers in almost all cases. This incision usually falls just at the dome of the diaphragm, which may need to be retracted.

Using internal finger palpation, the seventh intercostal space is located inferiorly at the midaxillary line, and an entry site is created through which a left atrial sump is placed. The sump tip is extracted from the working port, and any soft tissue debris along the suction ports are cleared, after which it is allowed to retract back into the chest. Carbon dioxide is infused 


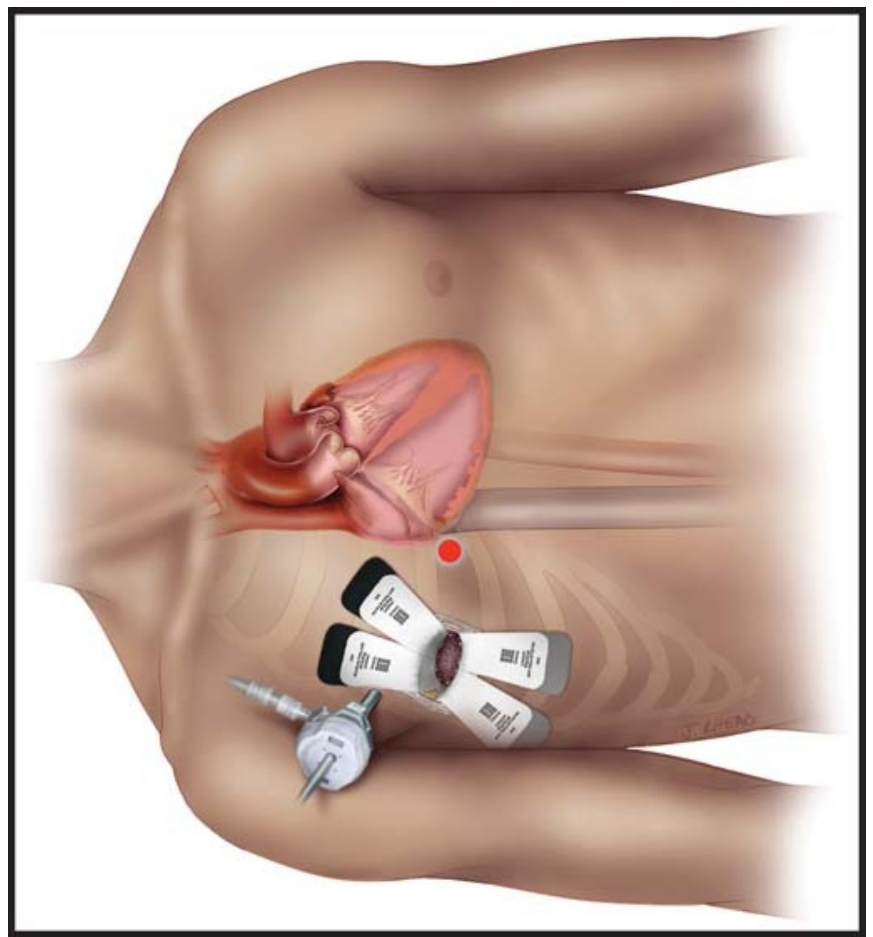

FIGURE 5. Incisions for working port and retractor. The red dot represents the location of the incision for the retractor.

in the field at a flow rate of 2 to $3 \mathrm{~L} / \mathrm{min}$ through a soft drain that can remain as a drainage tube.

A soft tissue retractor is inserted to retract the intercostal muscles, allowing for smooth introduction of long-shafted instruments and helping to preclude the inadvertent insertion of soft tissue debris during the intracardiac procedure.

Cannulation, myocardial protection, and de-airing are identical to those described in the direct-vision approach. The oblique sinus is opened widely, epicardial fat is cleared down to the atrial wall, and the Sondergaard groove is developed minimally to allow for accurate incision of the LA. A 5-mm trocar is placed through a separate stab wound one intercostal space above and more lateral than the working port, providing a "direct view" of the valve with a thoracoscope. A zero- or 30-degree thoracoscope should provide unimpeded lighting and visualization, and a table-mounted scope holder facilitates stabilization.

After aortic occlusion and cardioplegia arrest, a left atriotomy is performed and carried from the dome of the LA inferiorly through the oblique sinus, inferior to the right pulmonary veins. As discussed in the direct vision section, atrial wall "tenting" should be avoided. Internal finger palpation medially along the fourth intercostal space usually confirms proper placement of the atrial retractor blade post just lateral to the right sternal border. This minimizes the possibility of trauma to the internal thoracic artery and can be confirmed endoscopically before creating an entry site. The atrial retractor blade can now be delivered through the working port, connected to the post, and used to gently retract the interatrial septum anteriorly. A bedside stabilization device best affords stable fixation. Only minimal retraction is required, and the MV is rarely distorted (Fig. 6).

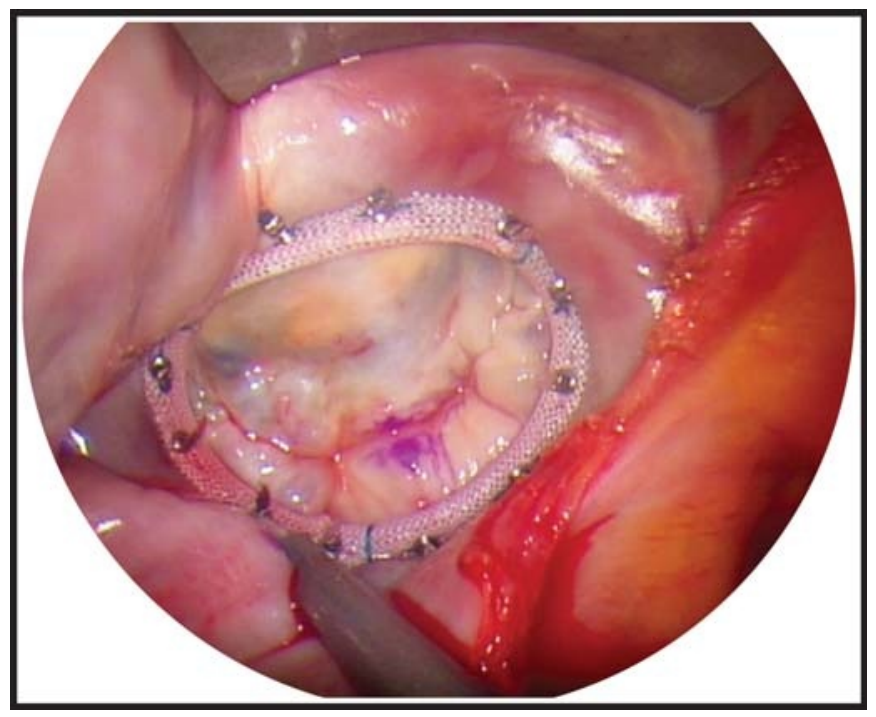

FIGURE 6. Retraction of the interatrial septum.

The previously inserted sump is now passed through the atriotomy and positioned within the left inferior pulmonary vein. The thoracoscope is advanced into the LA, and the remainder of the operation is performed videoscopically, with excellent visualization that allows a systematic, complete assessment of mitral leaflet morphology as well as the subvalvular apparatus (Fig. 7).

Long-shafted instruments are manipulated through the working port into the LA, allowing for any desired surgical technique according to surgeon preference. Direct visualization of the valve using this approach may be possible.

\section{SPECIAL CONSIDERATIONS}

\section{Transthoracic Cross-Clamping}

Aortic occlusion can be achieved with a transthoracic cross clamp or through endoaortic balloon occlusion, the comparative advantages and disadvantages of which are discussed in Ailadwadi et al. ${ }^{2}$ Early in their experience, most MIMVR

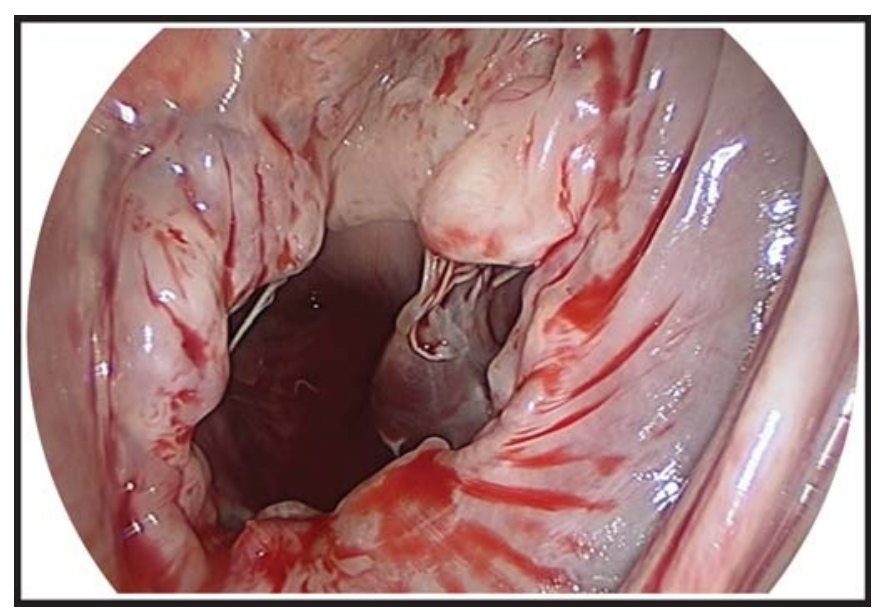

FIGURE 7. Endoscopic visualization of the MV. 
surgeons begin with cross clamping, which is familiar and allows direct clamping of the aorta as one would typically do through a sternotomy. This approach necessitates placement of a cardioplegia needle or catheter into the ascending aorta proximal to the cross clamp. Alternatively, a 14-gauge angiocatheter can be placed percutaneously along the sternum, taking care to avoid injury to the right internal mammary artery or intercostal vessels when traversing the chest wall. This technique is desirable in that the catheter is removed from the direct operative field; however, less optimal aortic venting may result owing to its long narrow design, and its flimsy nature may result in the catheter becoming kinked or completely dislodged. Repair of this site can facilitated by use of plegeted 2-0 polyester sutures and the use of an autoknotting device. As such, repair of this site is typically done while on pump to control aortic pressure while tying down sutures.

The clamp must be placed carefully in the transverse sinus, avoiding injury to the pulmonary artery and the left atrial appendage. If using a rigid clamp, kinking of the SVC can occur if significant atrial septal retraction is used. This can lead to restricted venous return unless the venous cannula is placed well into the SVC or a separate SVC cannula is used.

\section{Endoaortic Balloon Occlusion}

Use of endoaortic balloon (Edwards, Irvine, CA USA) occlusion presents its own unique set of benefits and challenges. ${ }^{2}$ While a comprehensive guide to the use of an endoaortic occlusion balloon is beyond the scope of this article, a brief description of its use and of the various forces acting on it may help surgeons in improving stability of the balloon within the aortic lumen.

The endoaortic balloon device is introduced through a hemostatic valved side arm on the arterial return catheter. It is positioned over a guidewire that has been previously advanced into the ascending aorta under either TEE or fluoroscopic guidance. The tip of the catheter is advanced until it is confirmed, by TEE, to be just above the sinotubular junction (STJ) as shown in Figure 8. The guidewire is then removed, leaving the central lumen for either cardioplegia administration or suction venting. Once the central lumen has been confirmed to be de-aired, both the balloon and root pressure tubing lines are connected to transducers and CPB is slowly instituted while a satisfactory and acceptable arterial-line perfusion pressure is confirmed.

There are numerous techniques of balloon deployment that allow for satisfactory and stable positioning. An occlusive endoaortic balloon will offer some resistance to migration by its radial force. Measuring the aortic diameter at the level of the STJ will provide a useful approximation of the necessary balloon volume required to provide stable occlusion. Balloon inflation with 1-mL saline per millimeter of aortic diameter will provide occlusion in most cases. This target volume is administered incrementally, anticipating an adequately inflated balloon pressure of 350 to $450 \mathrm{~mm} \mathrm{Hg}$. Overdistension with possible aortic trauma is to be avoided. A compliance/hysteresis change

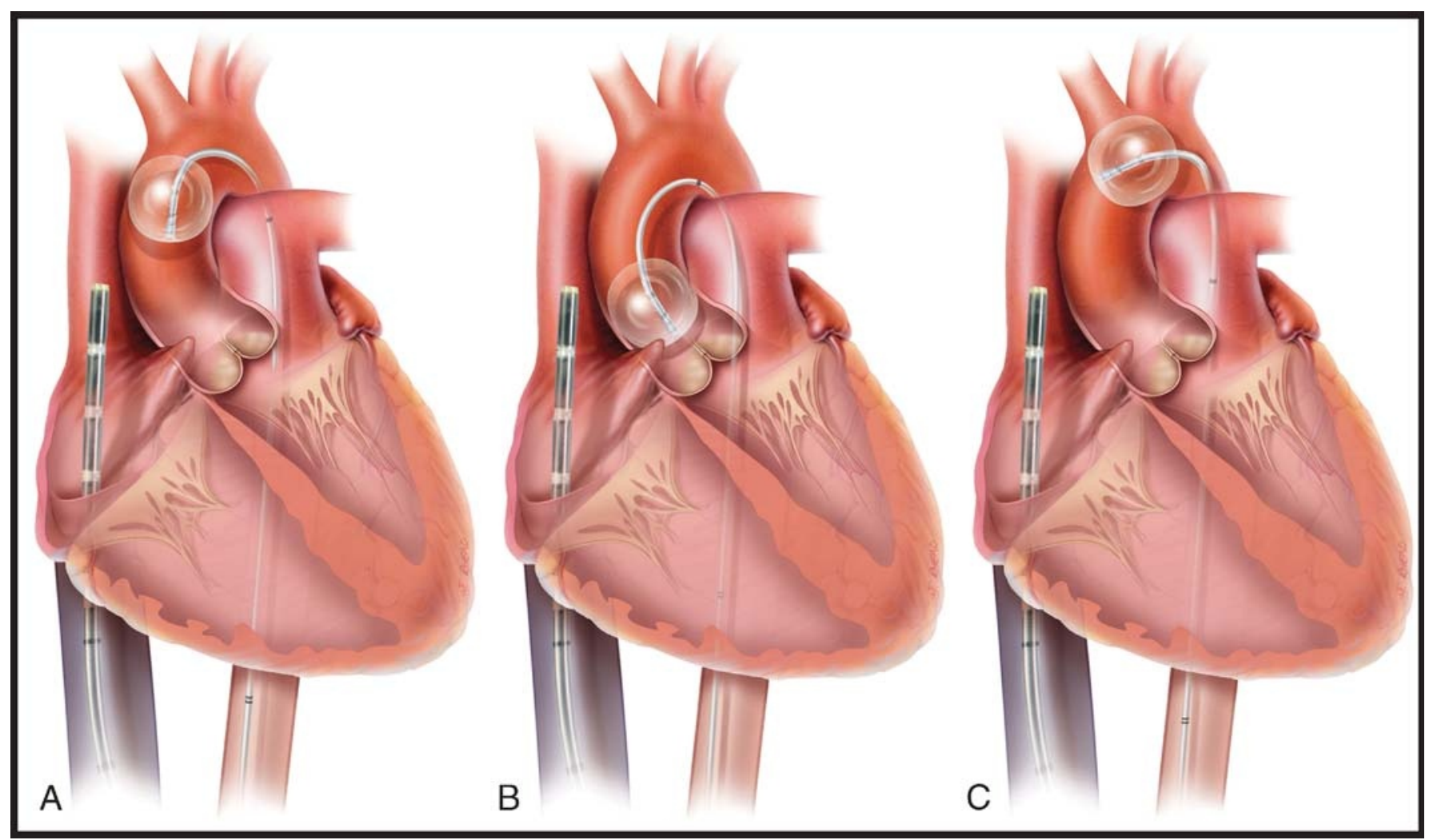

FIGURE 8. Endoaortic occlusion balloon positioning. A, Correct balloon position. B, Proximal migration may allow leakage around the balloon and possible iatrogenic puncture. C, Distal migration may diminish brachiocephalic perfusion. Note the tip of the venous cannula 2 to $3 \mathrm{~cm}$ above the junction of the right atrium and superior vena cava. 
within the wall of the balloon often results in a gradually decreasing inflation pressures to the 200 to 250 range, which should be ignored assuming a stable arrested heart. A properly inflated occlusive balloon will subsequently migrate according to the "relative forces" applied.

Emptying the venous circulation will mitigate balloon migration during initial inflation. The diminished ventricular stroke volume allows for balloon inflation to proceed under TEE guidance, maintaining its position above the STJ while confirming that pressures in the bilateral arterial lines remain similar. Once target inflation pressures are obtained, CPB flow rates can be restored, whereas antegrade cardioplegia infused into the aortic root provides "balance" against the forces of increasing systemic perfusion pressure on the balloon. An initial dose of adenosine (20-30 mg) may be infused to promote asystole during initial balloon deployment. At this point, observation of a "gradient" developing between the aortic root and systemic perfusion pressures is further indirect evidence of effective balloon occlusion.

During and immediately after cardioplegic arrest, the most dominant force on the balloon is from the pressure of arterial perfusion, which will usually exceed aortic root pressure from the cardioplegia pump. Increased arterial perfusion pressure will promote migration toward the valve, potentially resulting in circumferential leakage or subsequent iatrogenic injury. Transesophageal echocardiogram will show migration of the endoaortic balloon toward the aortic valve as arterial pressure increases and should be compensated for by catheter retraction to maintain the rim of the endoaortic balloon above the STJ. Active aortic root venting also will promote migration toward the valve. After cessation of antegrade cardioplegia, and certainly once the heart is open, systemic arterial perfusion pressure and catheter retraction are the dominant forces and must be balanced before final positioning. Once the balloon catheter is "locked" into position, it is imperative that arterial perfusion be maintained at relatively constant pressures.

Decreased arterial perfusion promotes migration away from the valve, which could lead to innominate artery occlusion and decreased right brachiocephalic blood flow. Subsequent dosing of antegrade cardioplegia in the presence of a competent aortic valve may result in transient balloon migration toward the innominate artery that subsides once the cardioplegia infusion is complete. This rise in aortic root pressure is important to confirm that the aortic root anatomy and thus valve competence is maintained and not adversely affected by the atrial septal retractor. Intermittent dosing of retrograde cardioplegia should have no effect on balloon position.

\section{Femoral Arterial Cannulation and Avoidance of Ischemic Leg Injury in MIMVR}

Ischemic injury to the leg is a documented potential complication of femoral arterial cannulation. The proposed mechanisms for this injury include misidentification of the common femoral artery, cannulation of a small femoral system, excessive perfusion times, unidentified vascular disease within this arterial system, and vascular injury or narrowing after removal of the cannula. In the patient without significant vascular disease, clamping or cannulating the common femoral artery should be reasonably well tolerated, with good backflow through the profunda system via collaterals. If the patient is cannulated either too close to the superficial femoral artery takeoff or if the superficial femoral artery is incorrectly identified as the common femoral artery, significant ischemic injury can occur. Compounding these issues is the relative obstruction of venous return caused by a concomitant cannulation of the ipsilateral femoral vein. These may lead to ischemic complications.

Avoidance of ischemic injury to the leg should be of primary concern for any minimally invasive approach. Careful preoperative screening (including $\mathrm{CT}$ angiography) and absolute certainty regarding cannulation of the common femoral vessel without occlusion of the femoral bifurcation should eliminate most if not all occurrences of this rare complication. Adjunctive precautions include measures to improve distal perfusion and monitoring for distal ischemia with near-infrared oximetry. Some centers use creation of a $\mathrm{T}$ graft using an 8-mm vascular graft sewn to the common femoral artery. This graft is then cannulated for arterial inflow, allowing blood to flow bidirectionally in the femoral system. Alternatively, distal (inferior) cannulation of the femoral artery is done with a small catheter (5-F arterial access sheath), using a short line taken off the arterial inflow.

\section{Postoperative Management}

Postoperative care should be tailored toward an accelerated recovery that takes advantage of the reduced surgical trauma associated with MIMVR. As previously noted, extubation in the operating room or within the first 6 hours is the goal and is facilitated by modified sedation and analgesia protocols. The keys to successful early extubation include an appropriate balance of sedation and analgesia to facilitate early extubation while allowing the patient to emerge with minimal incisional pain. A useful option for sedation is dexmedetomidine, which is unique in its ability to provide sedation without causing respiratory depression. Options for nonsedating parenteral analgesia include intravenous acetaminophen and intravenous ketorolac. Avoidance of long-term muscle relaxants during the procedure and complete rewarming of the patient will also facilitate early extubation.

After extubation, the benefits of adequate local pain control instituted in the operating room become apparent. A longacting intercostal block should routinely be administered before closure and options include bupivacaine, which has duration of approximately 6 hours, and liposomal bupivacaine, which can last up to 72 hours. Reservoir devices that allow continuous delivery of local anesthetic agents are also beneficial. Finally, cryoanalgesia is another adjunct for local analgesia and can be performed by freezing the intercostal nerve using a cryothermy probe before chest closure. ${ }^{16}$

Standard postoperative cardiac care applies for the MIMVR patient. Atrial fibrillation is common after cardiotomy and is treated per protocol. Prophylactic amiodarone is another strategy to reduce the rate of new-onset atrial fibrillation. ${ }^{17}$ Chest tubes are removed when drainage is less than $100 \mathrm{~mL}$ over 8 hours and serosanguinous. The goal is removal of all chest tubes, invasive lines, urinary catheter, and pacing wires by postoperative day 1. Aggressive diuresis should be maintained for 3 to 5 days while in the hospital, and this may reduce the risk of recurrent pleural effusions, which is a common reason for 
readmission. Patients should have the expectation of discharge by postoperative day 3 or 4 .

Standard sternal wound precautions are unnecessary for thoracotomy patients. Physical restrictions on upper extremity range of motion are relaxed upon discharge, and heavy lifting is titrated to pain tolerance. Patients may resume driving once they have ceased taking oral narcotics. No physical restrictions apply after 2 weeks.

\section{CONCLUSIONS}

Three established methods of performing MIMVR have been described. These include lower hemisternotomy, direct-vision right minithoracotomy, and endoscopic right minithoracotomy approaches. By consensus, a group of experienced surgeons has outlined safe methods for performing these procedures and a method of postoperative management that provides an accelerated recovery.

\section{REFERENCES}

1. Cohn LH, Adams DH, Couper GS, et al. Minimally invasive cardiac valve surgery improves patient satisfaction while reducing costs of cardiac valve replacement and repair. Ann Surg. 1997;226:421-426.

2. Ailawadi G, Agnihotri AK, Mehall JR, et al. Minimally invasive mitral valve surgery I: patient selection, evaluation, and planning. Innovations. 2016;11:243-250.

3. Lehr EJ, Guy TS, Smith RL, et al. Minimally invasive mitral valve surgery III: training and robotic-assisted approaches. Innovations. 2016;11:260-267.

4. Youssef SJ, Millan JA, Youssef GM, Earnheart A, Lehr EJ, Barnhart GR. The role of computed tomography angiography in patients undergoing evaluation for minimally invasive cardiac surgery: an early program experience. Innovations. 2015;10:33-38.

5. Rodriguez R, Gojkovic O, Khitri A, et al. Totally endoscopic minimally invasive mitral valve surgery as a routine approach: a 10 year experience. $\mathrm{Mi}$ tral Conclave American Association for Thoracic Surgery, Sheraton Towers, New York, NY USA, May 5-6, 2011.
6. Vanermen H, Farhat F, Wellens F, et al. Minimally invasive video-assisted mitral valve surgery: from Port-Access towards a totally endoscopic procedure. J Card Surg. 2000;15:51-60.

7. McClure RS, Cohn LH, Wiegerinck E, et al. Early and late outcomes in minimally invasive mitral valve repair: an eleven-year experience in 707 patients. J Thorac Cardiovasc Surg. 2009;137:70-75.

8. Ward AF, Grossi EA, Galloway AC. Minimally invasive mitral surgery through right mini-thoracotomy under direct vision. J Thorac Dis. 2013; 5(Suppl 6):S673-S679.

9. Lucà $\mathrm{F}$, van Garsse $\mathrm{L}$, Rao $\mathrm{CM}$, et al. Minimally invasive mitral valve surgery: a systematic review. Minim Invasive Surg. 2013;2013:179569.

10. Ritwick B, Chaudhuri K, Crouch G, Edwards JR, Worthington M, Stuklis RG. Minimally invasive mitral valve procedures: the current state. Minim Invasive Surg. 2013;2013:679276.

11. Vollroth M, Seeburger J, Garbade J, et al. Minimally invasive mitral valve surgery is a very safe procedure with very low rates of conversion to full sternotomy. Eur J Cardiothorac Surg. 2012;42:e13-e15.

12. Holzhey DM, Shi W, Borger MA, et al. Minimally invasive versus sternotomy approach for mitral valve surgery in patients greater than 70 years old: a propensity-matched comparison. Ann Thorac Surg. 2011;91:401-405.

13. Falk V, Cheng DC, Martin J, et al. Minimally invasive versus open mitral valve surgery: a consensus statement of the international society of minimally invasive coronary surgery (ISMICS) 2010. Innovations. 2011;6:66-76.

14. Svensson LG, Atik FA, Cosgrove DM, et al. Minimally invasive versus conventional mitral valve surgery: a propensity-matched comparison. $J$ Thorac Cardiovasc Surg. 2010;139:926-932.

15. Selman MW, McAlpine WA, Albregt H, Tatan R. An effective method of replacing air in the chest with $\mathrm{CO} 2$ during open-heart surgery. $J$ Thorac Cardiovasc Surg. 1967;53:618-622.

16. Pastor J, Morales P, Cases E, et al. Evaluation of intercostal cryoanalgesia versus conventional analgesia in postthoracotomy pain. Respiration. 1996;63: 241-245

17. Guarnieri T, Nolan S, Gottlieb SO, Dudek A, Lowry DR. Intravenous amiodarone for the prevention of atrial fibrillation after open heart surgery: the Amiodarone Reduction in Coronary Heart (ARCH) trial. J Am Coll Cardiol. 1999;34:343-347. 